\title{
Dihedral Rigidity of Parabolic Polyhedrons in Hyperbolic Spaces
}

\section{Chao LI}

Department of Mathematics, Princeton University, Fine Hall, 304 Washington Rd, Princeton, NJ 08544, USA

E-mail: chaoli@math.princeton.edu

URL: https://web.math.princeton.edu/ chaoli/

Received July 27, 2020, in final form September 30, 2020; Published online October 06, 2020

https://doi.org/10.3842/SIGMA.2020.099

\begin{abstract}
In this note, we establish the dihedral rigidity phenomenon for a collection of parabolic polyhedrons enclosed by horospheres in hyperbolic manifolds, extending Gromov's comparison theory to metrics with negative scalar curvature lower bounds. Our result is a localization of the positive mass theorem for asymptotically hyperbolic manifolds. We also motivate and formulate some open questions concerning related rigidity phenomenon and convergence of metrics with scalar curvature lower bounds.
\end{abstract}

Key words: dihedral rigidity; scalar curvature; comparison theorem; hyperbolic manifolds

2020 Mathematics Subject Classification: 53C21; 53A10

Dedicate this paper to Professor Misha Gromov on the occasion of his 75th birthday.

\section{Introduction}

In [14], Gromov proposed a geometric comparison theory for metrics with scalar curvature lower bounds. He speculated that Riemannian polyhedrons should play the role of triangles in Alexandrov's comparison theory for sectional curvature [1]. As a first step, he obtained the following theorem for metrics with nonnegative scalar curvature, where the comparison models are Euclidean cubes:

Theorem $1.1([14])$. Let $M=[0,1]^{n}$ be a cube, $g$ a smooth Riemannian metric. Then $(M, g)$ cannot simultaneously satisfy:

1) the scalar curvature $R(g) \geq 0$;

2) each face of $M$ is weakly strictly mean convex; ${ }^{1}$

3) the dihedral angles between adjacent faces are all acute.

Theorem 1.1 also has a rigidity statement: if $n \leq 7$, and we assume all dihedral angles are not larger than $\pi / 2$ in condition $(3)$, then $(M, g)$ is isometric to an Euclidean rectangular solid (see $[22,23])$. This is called the dihedral rigidity phenomenon. In [14, 15], Gromov conjectured that this property is satisfied for all convex polyhedrons in $\mathbf{R}^{n}$ :

This paper is a contribution to the Special Issue on Scalar and Ricci Curvature in honor of Misha Gromov on his 75th Birthday. The full collection is available at https://www.emis.de/journals/SIGMA/Gromov.html

${ }^{1}$ In this paper, the mean curvature is taken with respect to outer unit normal vector. For instance, the standard sphere $S^{n-1}$ in $\mathbf{R}^{n}$ has mean curvature $n-1$. 
Conjecture 1.2 (the dihedral rigidity conjecture). Let $M \subset \mathbf{R}^{n}$ be a convex polyhedron and $g_{0}$ be the Euclidean metric. Suppose $g$ is a smooth Riemannian metric on $M$. Denote its faces by $F_{i}$, the mean curvature of $F_{i}$ by $H_{i}$, and the dihedral angle between two adjacent faces $F_{i}, F_{j}$ by $\measuredangle_{i j}\left(\measuredangle_{i j}(g)\right.$ may be nonconstant $)$. Assume:

1) $R(g) \geq 0$ in $M$;

2) $H_{i}(g) \geq 0$ on each face $F_{i}$;

3) $\measuredangle_{i j}(g) \leq \measuredangle_{i j}\left(g_{0}\right)$ on each pair of adjacent faces $F_{i}, F_{j}$.

Then $(M, g)$ is isometric to a flat polyhedron in $\mathbf{R}^{n}$.

Conjecture 1.2 and related problems have been studied and extended in recent years (see, e.g., $[16,17,18,22,23,24,25])$, leading to a range of interesting new discoveries and questions on manifolds with nonnegative scalar curvature. In this paper, we investigate the analogous polyhedral comparison principle, together with the rigidity phenomenon, for metrics with negative scalar curvature lower bound.

By scaling, we assume $R(g) \geq-n(n-1)$. Our comparison model is a collection of polyhedrons in the hyperbolic space, called parabolic prisms, which we define now. Let $\left(\mathbf{H}^{n}, g_{H}\right)$ be the hyperbolic space with sectional curvature -1 . We choose the coordinate system $\left\{x_{1}, \ldots, x_{n}\right\}$, $x_{j} \in \mathbf{R}$, such that $g_{H}$ takes the form

$$
g_{H}=\mathrm{d} x_{1}^{2}+\mathrm{e}^{2 x_{1}}\left(\mathrm{~d} x_{2}^{2}+\cdots+\mathrm{d} x_{n}^{2}\right) .
$$

For any constant $c$, the coordinate hyperplane $x_{1}=c$ is umbilical with constant mean curvature $n-1$ with respect to $\partial_{x_{1}}$. The induced metric on it is isometrically Euclidean. These hyperplanes are called horospheres. For $j \geq 2$, the coordinate hyperplanes $x_{j}=c$ are totally geodesic, and they intersect each other and the horospheres orthogonally.

Denote $\hat{x}=\left(x_{2}, \ldots, x_{n}\right)$. Given a polyhedron $P \subset \mathbf{R}^{n-1}$, we call the set $\left\{\left(x_{1}, \hat{x}\right): 0 \leq\right.$ $\left.x_{1} \leq 1, \hat{x} \in P\right\}$ a parabolic prism in $\mathbf{H}^{n}$. As a special case of the main theorem of this paper, Theorem 2.4, we have the following

Theorem 1.3. Let $n \leq 7, M=[0,1]^{n}$ be a parabolic rectangle in $\mathbf{H}^{n}, g_{H}$ be the hyperbolic metric on $M$. Denote the face $\partial M \cap\left\{x_{1}=1\right\}$ by $F_{T}$, the face $\partial M \cap\left\{x_{1}=0\right\}$ by $F_{B}$. Assume $g$ is a Riemannian metric on $M$ such that:

1) $R(g) \geq-n(n-1)$ in $M$;

2) $H(g) \geq n-1$ on $F_{T}, H(g) \geq-(n-1)$ on $F_{B}$, and $H(g) \geq 0$ on $\partial M \backslash\left(F_{T} \cup F_{B}\right)$;

3 ) the dihedral angles between adjacent faces of $M$ are everywhere not larger than $\pi / 2$.

Then $(M, g)$ is isometric to a parabolic rectangle in $\mathbf{H}^{n}$.

Theorem 1.3 addresses a question discussed during the workshop "Emerging Topics on Scalar Curvature and Convergence" at IAS in October, 2018. See [17, Section 6].

We remark that Theorem 1.3 holds for any general Riemannian polyhedron $(M, g)$ with a proper polyhedral map to the parabolic cube of nonzero degree. It also holds for more general polyhedral types, as long as the comparison model is a parabolic prism $[0,1] \times P$, and $P \subset \mathbf{R}^{n-1}$ satisfies Conjecture 1.2. By [22, 23], $P$ can be any 3 -dimension simplices or $n$-dimensional non-obtuse prisms. See Theorem 2.3.

It has been observed in [23, Section 5] that Conjecture 1.2 is a localization of the positive mass theorem for asymptotically flat manifolds [27, 33]. Analogously, Theorem 1.3 localizes the positive mass theorem for asymptotically hyperbolic manifolds (see, e.g., [10, 11, 32]), which, in special cases, can be deduced from the following rigidity result of scalar curvature, due to Min-Oo [26] on spin manifolds and to Andersson-Cai-Galloway [2] on all manifolds of dimension at most 7 (see also $[9,21]$ for more recent developments): 
Theorem 1.4 ([2]; see also [26] for spin manifolds). Suppose $\left(S^{n}, g\right), 2 \leq n \leq 7$, has scalar curvature $R(g) \geq-n(n-1)$, and is isometric to $\mathbf{H}^{n}$ outside a compact set. Then $\left(S^{n}, g\right)$ is isometric to $\left(\mathbf{H}^{n}, g_{H}\right)$.

Precisely, suppose Theorem 1.3 holds. Given $\left(S^{n}, g\right)$ with $R(g) \geq-n(n-1)$ such that $(S, g)$ is isometric to $\left(\mathbf{H}^{n}, g_{H}\right)$ outside a compact set $K$, take a sufficiently large $R$ such that the boundary of the parabolic rectangle $M=[-R, R]^{n}$ isometrically embeds into $S \backslash K$. Denote the region bounded by $\partial M$ in $S$ by $M_{1}$. Then $M_{1}$ has a degree one map to $M$, by sending $K$ to an interior point $p \in M$ and $M_{1} \backslash K$ to $M \backslash\{p\}$. Thus Theorem 1.3 implies that $\left(M_{1}, g\right)$ is isometric to $\left(M, g_{H}\right)$.

Given the connection between the positive mass theorem and the dihedral rigidity conjecture, it would be interesting to see whether a similar comparison principle holds for metrics with positive scalar curvature lower bound. The delicate issue is that the corresponding rigidity phenomenon on a hemisphere is false, due to the counterexamples by Brendle-Marques-Neves [5]. This, together with other related open questions, will be discussed in Section 4 .

\section{Notations and the main theorem}

The main objects in this paper are Riemannian polyhedrons, which we define as follows.

Definition 2.1. A compact Riemannian manifold $\left(M^{n}, g\right)$ with boundary is called a Riemannian polyhedron, if $(M, g)$ can be isometrically embedded into $\mathbf{R}^{N}$ for some $N \geq n$, and at every $x \in M$, there is a radius $r>0$ and a diffeomorphism $\phi_{x}: B_{r}\left(x \in \mathbf{R}^{N}\right) \rightarrow B_{1}\left(0^{N}\right)$, such that $\phi_{x}\left(B_{r} \cap M\right)=P \cap B_{1}\left(0^{N}\right)$ for some Euclidean polyhedral cone $P$ of dimension $n$, and $\left.D \phi_{x}\right|_{x}$ is an isometry. Further, we require that $\phi_{x}$ is $C^{2, \alpha}$ for some $\alpha \in(0,1)$ independent of $x$.

Specially, a compact domain $M$ enclosed by piecewise $C^{2, \alpha}$ hypersurfaces in a smooth Riemannian manifold is a Riemannian polyhedron. Given $x \in M^{n}$, there is an integer $k \in[0, n]$ such that a neighborhood of $x$ in $M$ is diffeomorphic to $P_{0}^{n-k} \times \mathbf{R}^{k}$, and $P_{0}$ is a polyhedral cone in $\mathbf{R}^{n-k}$ without translation symmetry. We call the union of all such points the $k$-faces of $M$. In particular, the $n$-face is the interior of $M$, the $(n-1)$-faces are the union of smooth components of $\partial M$ (which we called "faces" in Theorem 1.3), and the $(n-2)$-faces are the interior of edges of $M$.

Definition 2.2. Let $P \subset \mathbf{R}^{n}$ be a flat Euclidean polyhedron, and $\left(M^{n}, g\right)$ be a Riemannian polyhedron. We say $M$ is over- $P$-polyhedral, if $M$ admits a proper polyhedral map $\phi$ onto $P$ (i.e., $\phi$ maps any $k$-face of $M$ to a $k$-face of $P$ ), such that $\phi$ is of nonzero degree.

In [22] and [23], Conjecture 1.2 was proved for a Riemannian polyhedrons that are over-Ppolyhedral, where

1) either $n=3$, and $P \subset \mathbf{R}^{3}$ is an arbitrary simplex;

2 ) or $3 \leq n \leq 7$, and $P$ is the Cartesian product $P_{0}^{2} \times[0,1]^{n-2}$. Here $P_{0} \subset \mathbf{R}^{2}$ is a polygon with non-obtuse dihedral angles.

Precisely, we have:

Theorem 2.3 ([22, 23]). Let $P \subset \mathbf{R}^{n}$ be as above, $\left(M^{n}, g\right)$ be an over-P-polyhedral Riemannian polyhedron, and $\phi: M \rightarrow P$ be the polyhedral map of nonzero degree. Suppose:

1) $R(g) \geq 0$ in $M$;

2) $H(g) \geq 0$ on each face of $M$; 
3) $\left.\measuredangle_{i j}(g)\right|_{x} \leq\left.\measuredangle_{i j}\left(g_{0}\right)\right|_{\phi(x)}$ for every point $x$ on the edges of $M$.

Then $(M, g)$ is isometric to an Euclidean polyhedron.

We now state the main theorem of this paper.

Theorem 2.4. Let $2 \leq n \leq 7, P \subset \mathbf{R}^{n-1}$ be an Euclidean polyhedron such that Theorem 2.3 holds for $P$. Denote $\left([0,1] \times P, g_{H}\right)$ the parabolic prism in the hyperbolic space with $R(g)=$ $-n(n-1)$. Suppose $\left(M^{n}, g\right)$ is a Riemannian polyhedron that is over-[0,1] $\times P$-polyhedral, and $\phi: M^{n} \rightarrow[0,1] \times P$ be the polyhedral map of nonzero degree. Suppose:

1) $R(g) \geq-n(n-1)$ in $M$;

2) $H(g) \geq n-1$ on $\phi^{-1}(\{1\} \times P), H(g) \geq-(n-1)$ on $\phi^{-1}(\{0\} \times P)$, and $H(g) \geq 0$ on other faces of $M$;

3) $\left.\measuredangle_{i j}(g)\right|_{x} \leq\left.\measuredangle_{i j}\left(g_{H}\right)\right|_{\phi(x)}$ for every point $x$ on the edges of $M$.

Then $(M, g)$ is isometric to a parabolic prism in the hyperbolic space.

Remark 2.5. The dimension restriction $n \leq 7$ in Theorems 2.3 and 2.4 is due to regularity of free boundary area minimizing surfaces and isoperimetric regions. In light of the recent progress on positive mass theorem in higher dimensions [29], we speculate the singular analysis may be applicable a non-rigid variance of Theorem 2.4, i.e., Theorem 1.1.

Remark 2.6. The condition that $P=P_{0} \times \mathbf{R}^{n-2}, P_{0}$ is non-obtuse, is due to the boundary regularity theory developed by Edelen and the author [13]. In general, one can guarantee that free boundary area minimizing surfaces are $C^{2, \alpha}$ regular in a non-obtuse polyhedral domain. See [13, Section 9].

\section{Proof of the main theorem}

The proof of Theorem 2.4 is an adaptation of the proof of Theorem 2.3. We will be using lots of techniques developed in [23]. Given a Riemannian polyhedron $\left(M^{n}, g\right)$ as in Theorem 2.4, denote the faces $F_{T}=\phi^{-1}(\{1\} \times P)$ and $F_{B}=\phi^{-1}(\{0\} \times P)$. If there exists a point $x$ on the edge of $M$ such that $\left.\measuredangle(g)\right|_{x}<\left.\measuredangle\left(g_{H}\right)\right|_{\phi(x)}$, we deform the metric $g$ to $\tilde{g}$ as in [16, Section 11], such that $\left.\measuredangle(\tilde{g})\right|_{x}=\left.\measuredangle\left(g_{H}\right)\right|_{\phi(x)}$ in a neighborhood of $x$ and the mean curvature of the two faces containing $x$ increases. Thus, without loss of generality, we assume that $\left.\measuredangle(g)\right|_{x}=\left.\measuredangle\left(g_{H}\right)\right|_{\phi(x)}$ for all points $x$ on the edge.

Consider the relative isoperimetric problem:

$$
\begin{gathered}
I=\inf \left\{\mathcal { H } ^ { n - 1 } \left(\partial \Omega\llcorner\stackrel{\circ}{M})-(n-1) \mathcal{H}^{n}(\Omega): \Omega \subset M\right.\right. \text { is a Caccioppoli set, } \\
\left.F_{B} \subset \Omega, F_{T} \cap \Omega=\varnothing\right\} .
\end{gathered}
$$

It follows from the standard compactness results that $I$ is achieved by a Caccioppoli set $\Omega$. Denote $\Sigma=\operatorname{spt}\left(\partial \Omega\llcorner\stackrel{\circ}{M})\right.$. Since $F_{B}, F_{T}$ meet other faces orthogonally and $H(g) \geq n-1$ on $F_{T}$, $H(g) \geq-(n-1)$ on $F_{B}$, by the strong maximum principle (see [23, Section 3.1]), either $\Sigma$ is disjoint from $F_{T}$ and $F_{B}$, or $\Sigma$ coincides with $F_{T}$ or $F_{B}$. In any case, $\Sigma$ is an isoperimetric surface with free boundary on $\partial M \backslash\left(F_{T} \cup F_{B}\right)$.

We remark here that similar variational problems as (3.1) have been considered by WittenYau [34] and by Andersson-Cai-Galloway [2], where it is called the BPS brane action.

We now study the regularity of $\Sigma$. Since $n \leq 7$, the regularity of $\Sigma$ in the interior and smooth part of $\partial M$ follows from the classical theory [20,30]. For a point $x \in \Sigma$ and a $k$-face of $M$ with $k \leq n-2$, we note that the tangent domain of $M$ at $x$ is given by $W^{2} \times[0, \infty)^{k-2} \times \mathbf{R}^{n-k}$, where $W^{2}$ is a wedge region in $\mathbf{R}^{2}$ with non-obtuse opening angle. Thus, we apply [13, Section 9] and [23, Appendix B], and conclude: 
Proposition 3.1. $\Sigma$ is $C^{2, \alpha}$ graphical over its tangent plane everywhere.

Moreover, since $\Sigma$ is homologous to $F_{B}$, we conclude that at least one connected component (which we still denote by $\Sigma$ ) has a nonzero degree map to $P$, given by $\Sigma \simeq F_{B} \stackrel{\phi}{\rightarrow}\{0\} \times P$.

Since $\Omega$ is a minimizer for (3.1), $\Sigma$ has constant mean curvature $(n-1)$ with respect to the outer unit normal $\nu$ of $\Omega$, and stability implies that

$$
\begin{aligned}
Q(\varphi):= & \int_{\Sigma}|\nabla \varphi|^{2}-\frac{1}{2}\left(R_{M}-R_{\Sigma}+n(n-1)+|\AA|^{2}\right) \varphi^{2} \mathrm{~d} \mathcal{H}^{n-1} \\
& -\int_{\partial \Sigma} \operatorname{II}(\nu, \nu) \varphi^{2} \mathrm{~d} \mathcal{H}^{n-2} \geq 0,
\end{aligned}
$$

for all $C^{1}$ function $\varphi$. Here $R_{\Sigma}, \AA$ are the scalar curvature of the induced metric and the traceless second fundamental of $\Sigma$, respectively, and II is the second fundamental form of $\partial M$.

Let $\varphi>0$ be the principal eigenfunction associated with the quadratic form $Q$. Then $\varphi$ solves the equation

$$
\left\{\begin{array}{l}
\Delta_{\Sigma} \varphi+\frac{1}{2}\left(R_{M}-R_{\Sigma}+n(n-1)+|\AA|^{2}\right) \varphi=-\lambda_{1} \varphi \\
\frac{\partial \varphi}{\partial \eta}=\operatorname{II}(\nu, \nu) \varphi .
\end{array}\right.
$$

Here $\eta$ is the outer conormal vector field of $\Sigma$, and $\lambda_{1}$ is the principal eigenvalue associated with $Q$. It follows from [23, Lemma 4.1] that $\varphi \in C^{2, \alpha}(\Sigma)$. Denote $\tilde{g}=\varphi^{\frac{2}{n-2}} g$ on $\Sigma$. By the very same calculations as in [23, equations (4.6) and (4.7)], we have

$$
R(\tilde{g})=\varphi^{-\frac{n}{n-2}}\left(\left(R_{M}+n(n-1)+|\stackrel{\AA}{2}|^{2}+\lambda_{1}\right) \varphi+\frac{n-1}{n-2} \frac{|\nabla \varphi|^{2}}{\varphi}\right) \geq 0,
$$

and $H_{\partial \Sigma}(\tilde{g})=\varphi^{-\frac{1}{n-2}}\left(H_{\partial \Sigma}(g)+\mathrm{II}(\nu, \nu)=\varphi^{-\frac{1}{n-2}} H_{\partial M}(g) \geq 0\right.$.

Moreover, since $\Sigma$ meets $\partial M$ orthogonally and $\tilde{g}$ is conformal to $g$, the dihedral angles of $(\Sigma, \tilde{g})$ is everywhere equal to that of $P$. Thus, by Theorem $2.3,(P, \tilde{g})$ is isometric to an Euclidean polyhedron. Tracing equality, we have

$$
R_{M}=0, \quad \AA=0, \quad \lambda_{1}=0, \quad \nabla \varphi=0 \quad \text { on } \Sigma .
$$

Therefore $\varphi$ is a constant function, and hence $\operatorname{Ric}_{M}(\nu, \nu)=-(n-1)$ on $\Sigma$ and $\operatorname{II}(\nu, \nu)=0$ on $\partial \Sigma$. It follows that $\Sigma$ is totally umbilical and infinitesimally rigid.

Next, we adapt the ideas in $[7,8]$ to study rigidity. Let $M^{-}$be the region enclosed by $\Sigma$ and $F_{B}$. We follow the same argument as in [23, Section 4]: by constructing the very same deformed metrics $\{g(t)\}_{t \in[0, \varepsilon)}$, solving the relative isoperimetric problem (3.1) and taking convergence as $t \rightarrow 0$, we obtain another free-boundary isoperimetric hypersurface $\Sigma^{\prime}$ in $(M, g)$ lying between $\Sigma$ and $F_{B}$. Moreover, $\Sigma^{\prime}$ is also isometrically Euclidean, and is umbilical and infinitesimally rigid. By repeating this argument, we obtain a dense collection of such hypersurfaces $\left\{\Sigma^{\rho}\right\}$ in $M$.

Fix $\Sigma^{\rho}$, its outer unit normal $\nu$, and $x_{0} \in \Sigma^{\rho}$. For $\rho_{j}$ sufficiently close to $\rho, \Sigma^{\rho_{j}}$ can be written as a normal graph of function $u^{j}$ over $\Sigma^{\rho}$. By standard curvature, the function $u^{j} / u^{j}\left(x_{0}\right)$ converges in $C^{2, \alpha}\left(\Sigma^{\rho}\right)$ to a nonzero function $u$. The Gauss-Codazzi equation implies that, for any tangential vector $X, Y$ on $\Sigma^{\rho}$,

$$
\left(\nabla_{\Sigma^{\rho}}^{2} u\right)(X, Y)+R m_{M}(\nu, X, Y, \nu) u+A_{\Sigma^{\rho}}(X, Y) u=0 .
$$

Taking trace, we have that $\Delta_{\Sigma^{\rho}} u=0$. Also, since $\Sigma^{\rho_{j}}$ meets $\partial M$ orthogonally and $\operatorname{II}(\nu, \nu)=0$ on $\partial \Sigma^{\rho_{j}}, \frac{\partial u}{\partial \eta}=0$. Thus $u$ is a constant function, and hence $\operatorname{Rm} m_{M}(\nu, X, Y, \nu)=-\langle X, Y\rangle$. This proves that $M$ has constant sectional curvature -1 . Theorem 2.4 is proved. 


\section{Discussions and related questions}

\subsection{Metrics with positive scalar curvature lower bounds}

It is tempting to conjecture that a suitable extension of Theorems 2.3 and 2.4 holds for metrics with positive scalar curvature lower bounds, where the model space is $S^{n}$ with a round metric. Although the precise formulation is unclear, such an extension will likely to localize certain scalar curvature rigidity phenomenon for spheres. Recall the following theorem by Brendle and Marques [4]:

Theorem $4.1([4])$. Let $\Omega=B(\delta) \subset S^{n}$ be a closed geodesic ball of radius $\delta$ with $\cos \delta \geq \frac{2}{\sqrt{n+3}}$, and $\bar{g}$ be the standard metric on $S^{n}$. Suppose $g$ is another metric on $\Omega$ such that:

1) $R(g) \geq R(\bar{g})$ in $\Omega$;

2) $H(g) \geq H(\bar{g})$ on $\partial \Omega$;

3) $g$ and $\bar{g}$ induce the same metric on $\partial \Omega$.

If $g-\bar{g}$ is sufficiently small in the $C^{2}$-norm, then $g$ is isometric to $\bar{g}$.

The lower bound $\frac{3}{\sqrt{n+3}}$ for $\delta$ was improved in a subsequent paper by Cox, Miao and Tam [12]. However, it is known that the analogous statement for $\delta=\frac{\pi}{2}$ does not hold, due to the counterexample by Brendle, Marques and Neves [5].

The original proof of Theorem 1.1 by Gromov uses the fact that a cube is the fundamental domain of $\mathbf{Z}^{n}$ action on $\mathbf{R}^{n}$ : assuming a counterexample for Theorem 1.1 exists, through a sequence of doubling and smoothing, one obtains a smooth metric on $T^{n}$ with positive scalar curvature, contradicting [19] and [28].

Take the standard embedding $S_{+}^{n} \subset \mathbf{R}^{n+1}$. The hemisphere $S_{+}^{n}=S^{n} \cap\left\{x_{n+1} \geq 0\right\}$ can be obtained by consecutive doublings of the spherical simplex

$$
\Omega_{n}:=S^{n} \cap\left\{x_{j} \geq 0, j=1, \ldots, n+1\right\} .
$$

We make the following conjecture concerning dihedral rigidity of $\Omega_{n}$.

Conjecture 4.2. Let $\left(M^{n}, g\right)$ be a Riemannian polyhedron which is diffeomorphic to a simplex of dimension n. Suppose

1) $R(g) \geq n(n-1)$ in $M$;

2) $\partial M$ is piecewise totally geodesic;

3) $\measuredangle_{i j}(g) \leq \frac{\pi}{2}$ on the edges of $M$;

4) moreover, each face of $M$ is globally isometric to the standard spherical simplex $\Omega_{n-1}$.

Then $\left(M^{n}, g\right)$ is isometric to $\Omega_{n}$.

When $n=2$, Conjecture 4.2 holds by doubling $M$ twice across its boundary, and using a theorem due to Toponogov [31]. On the other hand, the construction in [5] does not seem to give a counterexample to Conjecture 4.2 .

\subsection{Weak notions of $R \geq \kappa$}

One of Gromov's motivations of studying Conjecture 1.2 is to define the notion of " $R \geq 0$ " in the weak sense. The crucial observation is that, the conditions (2), (3) concern $C^{0}$ properties of the metric $g$, and are stable under $C^{0}$ convergence of metrics (see [14]). Thus, we may define

$$
\begin{aligned}
\text { "R } R(g) \geq 0 " \Leftrightarrow & \text { there exists no cube } M \\
& \text { with mean convex faces and everywhere acute dihedral angle. }
\end{aligned}
$$


And more generally for $\kappa<0$,

$$
\begin{aligned}
\text { "R(g) } \geq \kappa " \Leftrightarrow & \text { there exists no cube } M \text { in the product with } S_{-\kappa}^{2} \\
& \text { with mean convex faces and everywhere acute dihedral angle. }
\end{aligned}
$$

Here $S_{-\kappa}^{2}$ is the space form with scalar curvature $-\kappa$.

Using this observation, Gromov proved the following theorem on the convergence of metrics with scalar curvature lower bounds:

Theorem 4.3 ([14], see also [3]). Let $M^{n}$ be a smooth manifold, $g, g_{k}, k \geq 1$, be smooth Riemannian metrics on $M$, and $g_{k} \rightarrow g$ in $C^{0}$ as tensors. Suppose $R\left(g_{k}\right) \geq \kappa$ on $M$. Then $R(g) \geq \kappa$ as well.

Based on Theorem 2.4, we may define $R \geq \kappa$ for a negative constant $\kappa$ :

$$
\begin{aligned}
\text { "R(g) } \geq-\kappa " \Leftrightarrow & \text { there exists no cube } M \text { with acute dihedral angles } \\
& \text { and faces }\left\{F_{j}\right\} \text {, such that } H>-(n-1) \text { on } F_{1}, \\
& H>0 \text { on other faces, and } H>(n-1) \text { on the opposite face of } F_{1} .
\end{aligned}
$$

(4.1) and (4.2) should be equivalent on smooth metrics, but we think that (4.2) is sightly more natural conceptually, as it also satisfies the dihedral rigidity phenomenon.

Recently, Burkhardt-Guim [6] proposed a different possible notion of " $R>\kappa$ " using Ricci flow. See [6, Definition 1.2]. These definitions all share some good properties as a weak notion. For instance, they all agree to $R(g)>\kappa$ in the classical sense for a $C^{2}$ metric $g$, and they can be localized in a neighborhood of any point on the manifold. The natural question is:

Question 4.4. Given a smooth manifold $M$ and a $C^{0}$ metric $g$ on it. Do the definitions (4.2) and [6, Definition 1.2] agree on $g$ ?

\section{Acknowledgements}

I would like to thank Christina Sormani and Misha Gromov for organizing the excellent workshop "Emerging Topics on Scalar Curvature and Convergence" at the Institute for Advanced Study, and everyone in the workshop for valuable discussions. The author is supported by NSF grant DMS-2005287.

\section{References}

[1] Aleksandrov A.D., Berestovskii V.N., Nikolaev I.G., Generalized Riemannian spaces, Russian Math. Surveys 41 (1986), 1-54.

[2] Andersson L., Cai M., Galloway G.J., Rigidity and positivity of mass for asymptotically hyperbolic manifolds, Ann. Henri Poincaré 9 (2008), 1-33, arXiv:math.DG/0703259.

[3] Bamler R.H., A Ricci flow proof of a result by Gromov on lower bounds for scalar curvature, Math. Res. Lett. 23 (2016), 325-337, arXiv:1505.00088.

[4] Brendle S., Marques F.C., Scalar curvature rigidity of geodesic balls in $S^{n}$, J. Differential Geom. 88 (2011), 379-394, arXiv:1005.2782.

[5] Brendle S., Marques F.C., Neves A., Deformations of the hemisphere that increase scalar curvature, Invent. Math. 185 (2011), 175-197, arXiv:1004.3088.

[6] Burkhardt-Guim P., Pointwise lower scalar curvature bounds for $C^{0}$ metrics via regularizing Ricci flow, Geom. Funct. Anal. 29 (2019), 1703-1772, arXiv:1907.13116.

[7] Carlotto A., Chodosh O., Eichmair M., Effective versions of the positive mass theorem, Invent. Math. 206 (2016), 975-1016, arXiv:1503.05910. 
[8] Chodosh O., Eichmair M., Moraru V., A splitting theorem for scalar curvature, Comm. Pure Appl. Math. 72 (2019), 1231-1242, arXiv:1804.01751.

[9] Chruściel P.T., Delay E., The hyperbolic positive energy theorem, arXiv:1901.05263.

[10] Chruściel P.T., Herzlich M., The mass of asymptotically hyperbolic Riemannian manifolds, Pacific J. Math. 212 (2003), 231-264, arXiv:math.DG/0110035.

[11] Chruściel P.T., Nagy G., The mass of spacelike hypersurfaces in asymptotically anti-de Sitter space-times, Adv. Theor. Math. Phys. 5 (2001), 697-754, arXiv:gr-qc/0110014.

[12] Cox G., Miao P., Tam L.-F., Remarks on a scalar curvature rigidity theorem of Brendle and Marques, Asian J. Math. 17 (2013), 457-469, arXiv:1109.3942.

[13] Edelen N., Li C., Regularity of free boundary minimal surfaces in locally polyhedral domains, arXiv:2006.15441.

[14] Gromov M., Dirac and Plateau billiards in domains with corners, Cent. Eur. J. Math. 12 (2014), 1109-1156, arXiv:1811.04318.

[15] Gromov M., A dozen problems, questions and conjectures about positive scalar curvature, in Foundations of Mathematics and Physics One Century after Hilbert, Springer, Cham, 2018, 135-158, arXiv:1710.05946.

[16] Gromov M., Metric inequalities with scalar curvature, Geom. Funct. Anal. 28 (2018), 645-726, arXiv:1710.04655.

[17] Gromov M., Scalar curvature of manifolds with boundaries: natural questions and artificial constructions, arXiv:1811.04311.

[18] Gromov M., Four lectures on scalar curvature, arXiv:1908.10612.

[19] Gromov M., Lawson Jr. H.B., Spin and scalar curvature in the presence of a fundamental group. I, Ann. of Math. 111 (1980), 209-230.

[20] Grüter M., Jost J., Allard type regularity results for varifolds with free boundaries, Ann. Scuola Norm. Sup. Pisa Cl. Sci. (4) 13 (1986), 129-169.

[21] Huang L.-H., Jang H.C., Martin D., Mass rigidity for hyperbolic manifolds, Comm. Math. Phys. 376 (2020), 2329-2349, arXiv:1904.12010.

[22] Li C., A polyhedron comparison theorem for 3-manifolds with positive scalar curvature, Invent. Math. 219 (2020), 1-37, arXiv:1710.08067.

[23] Li C., The dihedral rigidity conjecture for $n$-prisms, arXiv:1907.03855.

[24] Li C., Mantoulidis C., Positive scalar curvature with skeleton singularities, Math. Ann. 374 (2019), 99-131, arXiv:1708.08211.

[25] Miao P., Measuring mass via coordinate cubes, Comm. Math. Phys. 379 (2020), 773-783, arXiv:1911.11757.

[26] Min-Oo M., Scalar curvature rigidity of asymptotically hyperbolic spin manifolds, Math. Ann. 285 (1989), 527-539.

[27] Schoen R., Yau S.-T., On the proof of the positive mass conjecture in general relativity, Comm. Math. Phys. 65 (1979), 45-76.

[28] Schoen R., Yau S.-T., On the structure of manifolds with positive scalar curvature, Manuscripta Math. 28 (1979), 159-183.

[29] Schoen R., Yau S.-T., Positive scalar curvature and minimal hypersurface singularities, arXiv:1704.05490.

[30] Simons J., Minimal varieties in riemannian manifolds, Ann. of Math. 88 (1968), 62-105.

[31] Toponogov V.A., Evaluation of the length of a closed geodesic on a convex surface, Dokl. Akad. Nauk SSSR 124 (1959), 282-284.

[32] Wang X., The mass of asymptotically hyperbolic manifolds, J. Differential Geom. 57 (2001), 273-299.

[33] Witten E., A new proof of the positive energy theorem, Comm. Math. Phys. 80 (1981), 381-402.

[34] Witten E., Yau S.-T., Connectedness of the boundary in the AdS/CFT correspondence, Adv. Theor. Math. Phys. 3 (1999), 1635-1655, arXiv:hep-th/9910245. 